\title{
Baseline demographic, clinical and laboratory risk factors for predicting admission to intensive care unit in patients diagnosed with COVID-19 in the emergency department
}

\author{
두릴t Güngörer \\ University of Health Sciences, Ankara City Hospital, Department of Emergency Medicine, Ankara, Turkey
}

Cite this article as: Güngörer B. Baseline demographic, clinical and laboratory risk factors for predicting admission to intensive care unit in patients diagnosed with COVID-19 in the emergency department. Anatolian Curr Med J 2021; 3(4); 279-283.

\begin{abstract}
Aim: The COVID-19 pandemic has caused very significant morbidity and mortality throughout the world. Predicting the need for intensive care in these patients is important in terms of proper planning of health services and developing cost-effective management strategies. In this study, we sough to investigate the predictability of whether patients with COVID-19 would need intensive care by looking at some clinical, hematological and biochemical parameters.

Material and Method: All of the patients who applied to the adult emergency department of our hospital with the diagnosis of COVID-19 and were hospitalized were included in the study. The age, physical examination findings, comorbidities, and first laboratory parameters of the patients admitted to our hospital between March 2020 and June 2020 were retrospectively analyzed. A multivarible logistic regression model was constructed to determine the significant predictors of admission to ICU.

Results: A total of 1,005 patients were included in the study. Logistic regression analyses revealed that age (OR: $1.094, \mathrm{p}<0.001)$, chronic renal failure (OR: 4.735, $\mathrm{p}=0.036)$, cancer diagnosis (OR: 3.957, $\mathrm{p}=0.021)$, higher levels of lactate dehydrogenase (OR: 1.006 , $\mathrm{p}<0.001$ ), and ferritin (OR: 1.001, $\mathrm{p}=0.001$ ), and lower levels of lymphocyte count (OR: 0.879, $\mathrm{p}=0.021$ ) were the independent risk factors in predicting the intensive care unit admission of the patients

Conclusion: Age, chronic renal failure, cancer, higher levels of $\mathrm{LDH}$ and ferritin, and lower levels of lymphocyte are found to be independent risk factors in predicting intensive care admission for patients admitted to the emergency department with the diagnosis of COVID-19.
\end{abstract}

Keywords: COVID-19, emergency department, intensive care unit

\section{INTRODUCTION}

The coronavirus 2019 disease (COVID 19) caused by a new strain of coronavirus, SARS-CoV 2, has caused a pandemic. It led to millions of people being hospitalized and being hospitalised in intensive care unit $(1,2)$. For a long time, health systems caused serious problems in many countries due to lack of space and intensive care beds, and people were unable to access health services. Emergency services became the most important actor of this pandemic and had to decide which patients will have a poor prognosis and which patients will be treated as outpatients, and doing this in a very short time has become an important approach for the management of patients (3). In some studies, clinical clues such as the age of the patients, underlying diseases, some hematological parameters and radiological findings were investigated whether the patients should be hospitalized and whether they needed intensive care $(4,5)$. During the pandemic in Turkey, especially large hospitals were accepted as pandemic hospitals and took an active role in the management of the pandemic. In this regard, ${ }^{* *}$ has become a very important center in Turkey with the number of patients admitted, the number of beds and the number of intensive care units. We planned this retrospective study with the thought that with the decrease in the number of applications today, there are clues about which patients will have a poor prognosis and which patients may need intensive care, and it will be of great help to the clinician. With this research, we investigated the predictability of whether patients with COVID-19 would need intensive care by looking at some clinical, hematological and biochemical parameters. 


\section{MATERIAL AND METHOD}

The study was carried out with the permission of Ankara City Hospital No. 2 Clinical Researchs Ethics Committee (Date: 14.07.2021, Decision No: E2-21-739). All procedures were carried out in accordance with the ethical rules and the principles of the Declaration of Helsinki.

\section{Patients}

All of the patients who applied to the adult emergency department with the diagnosis of COVID-19 and were hospitalized were included in the study. The ages, examination findings, comorbidities, and first laboratory parameters of the patients admitted between March 2020 and June 2020 were retrospectively analyzed. All hospitalized patients older than 18 years with positive polymerase chain reaction (PCR) tests were included. Patients whose laboratory findings were missing and clinical information could not be reached were excluded from the study. The patients were separated into two groups as those admitted to the normal service and those requiring intensive care, and compared in terms of these parameters. The patients' ages, pregnancy status, presence of hypertension, diabetes, coronary artery disease, cronic obstructive pulmonary disease, chronic kidney failure, cancer, immunodeficiency, pneumonia, and whether the patient was a healthcare worker were recorded. Hematological data of the patients, hemoglobin levels, white blood cell counts, lymphocyte count, platelet count were obtained from the hospital data system. Among the biochemical parameters, creatinine level, sodium, potassium, lactate dehydrogenase, erythrocyte sedimentation rate, $\mathrm{c}$ reactive protein, interleukin 6 level, procalcitonin level, ferritin, alanine aminotransferase, aspartate aminotransferase, alkaline phosphatase and troponin levels were recorded on patient follow-up forms.

Admission to intensive care unit was done according to current guidelines (6). Severe illness, severe respiratory tract infection (severe pneumonia; respiratory rate $\geq 30$ / min and/or severe respiratory distress (dyspnea, use of extra respiratory muscles) and/or oxygen saturation in room air $\leq 90 \%$ ), hypotension (systolic blood pressure $<90 \mathrm{mmHg}$ and more than $40 \mathrm{mmHg}$ decrease from normal systolic blood pressure and mean arterial pressure $<65 \mathrm{mmHg}$, tachycardia >100/min, troponin elevation, presence of skin disorders such as capillary return disorder and cutis marmaratus, acute respiratory distress dyndrome, sepsis, septic shock, myocarditis, arrhythmia and cardiogenic shock, metabolic acidosis, coagulation disorder and multiple organ failure.

\section{Statistical Analysis}

All statistical analysis entered to Stata computer based programme (version16.0 MP; StataCorp). The distribution of continuous variables was determined using Kolmogorov-Smirnov. Of the continuous data, those with normal distribution were presented as mean \pm standard deviation (SD), and data without normal distribution were presented as median (range). Categorical data were defined as the number of cases. Statistical analysis differences in variables those with normal distribution were compared.

Student's t-test was used for two different groups (ICU and non-ICU patients) with normal distribution, MannWhitney U test was used for data not normally distributed. Categorical variables were compared using Pearson's Chi square test. A univariate logistic regression model was constructed for each variable to show significant predictors of ICU admissions, and then those with $\mathrm{p}<0.1$ values were tested in the multivariate logistic regression model. Odds ratios (ORs) and their 95\% confidence intervals (CI) for ICU admission are presented. $\mathrm{p}<0.05$ was considered significant in all statistical analyses.

\section{RESULTS}

A total of 1,005 patients were included in the study. While 847 patients were admitted to the normal inpatient service, 158 of them were hospitalised to the intensive care unit. The mean age of the patients admitted to the intensive care unit was higher than the patients admitted to the normal service $(42.9 \pm 15.7$ vs $67.3 \pm 14.5, \mathrm{p}<0.001)$. There was no difference between the groups in terms of gender and pregnancy. Hypertension, diabetes, chronic obstructive pulmonary disease, chronic kidney failure, cancer, pneumonia and being a healthcare worker differed significantly between the groups. These data are summarized in Table 1.

When the laboratory data were compared between the two groups, it was seen that all other laboratory data were statistically different between the two groups, except for platelet, sodium and potassium levels. All these parameters are also summarized in Table 2.

Table 1. Baseline clinical and demographical findings of the study population

\begin{tabular}{|lccc|}
\hline & $\begin{array}{c}\text { Non-ICU } \\
(\mathbf{n}=\mathbf{8 4 7})\end{array}$ & $\begin{array}{c}\text { ICU } \\
(\mathbf{n}=158)\end{array}$ & p-value \\
\hline Age, mean (SD) & $42.9(15.7)$ & $67.3(14.5)$ & $<0.001$ \\
Male, n (\%) & $438(51.7 \%)$ & $88(55.7 \%)$ & 0.36 \\
Pregnancy, n (\%) & $16(9.8 \%)$ & $0(0.0 \%)$ & 0.083 \\
Hypertension, n (\%) & $79(9.3 \%)$ & $48(30.4 \%)$ & $<0.001$ \\
Diabetes mellitus, n (\%) & $56(6.6 \%)$ & $19(12.0 \%)$ & 0.017 \\
$\begin{array}{l}\text { Chronic obstructive } \\
\text { pulmonary disease, } \mathrm{n}(\%)\end{array}$ & $16(1.9 \%)$ & $18(11.4 \%)$ & $<0.001$ \\
$\begin{array}{l}\text { Coronary artery disease, } \\
\text { n (\%) }\end{array}$ & $11(1.3 \%)$ & $4(2.5 \%)$ & 0.24 \\
Chronic renal failure, n (\%) & $5(0.6 \%)$ & $7(4.4 \%)$ & $<0.001$ \\
Cancer, n (\%) & $9(1.1 \%)$ & $13(8.2 \%)$ & $<0.001$ \\
Immun deficiency, n (\%) & $2(0.2 \%)$ & $1(0.6 \%)$ & 0.40 \\
Pneumonia, n (\%) & $359(42.4 \%)$ & $88(55.7 \%)$ & 0.002 \\
Health-care workers, n (\%) & $44(5.2 \%)$ & $1(0.6 \%)$ & 0.011 \\
\hline
\end{tabular}




\begin{tabular}{|lccc|}
\hline Table 2. Initial laboratory findings of the study population & & & \\
\hline & Non-ICU (n=847) & ICU (n=158) & p-value \\
\hline Hemoglobin, mean (SD) & $13.8(1.7)$ & $12.7(2.1)$ & $<0.001$ \\
White blood cell, mean (SD) & $5.6(2.5)$ & $7.5(6.3)$ & $<0.001$ \\
Neutrophil, mean (SD) & $3.6(2.1)$ & $5.7(4.9)$ & $<.0 .001$ \\
Lymphocyte, median (IQR) & $1.4(1.0,1.9)$ & $0.9(0.5,1.2)$ & $<0.001$ \\
Platelet, mean (SD) & $226.8(80.4)$ & $1.1(0.7)$ & 0.23 \\
Creatinine, mean (SD) & $0.8(0.5)$ & $138.6(7.3)$ & $<0.001$ \\
Sodium, mean (SD) & $139.1(3.2)$ & $4.0(0.5)$ & 0.15 \\
Potassium, mean (SD) & $4.1(0.4)$ & $331.3(234.5,424.0)$ & 0.19 \\
Lactate dehydrogenase, median (IQR) & $214.0(181.8,270.5)$ & $86.9(25.9,165.0)$ & $<0.001$ \\
C-reactive protein, median (IQR) & $5.0(1.1,25.6)$ & $52.5(40.4)$ & $<0.001$ \\
Erythrocyte sedimentation rate, mean (SD) & $24.9(23.5)$ & $41.7(12.9,90.4)$ & $<0.001$ \\
Interleukin-6, median (IQR) & $8.1(1.1,37.9)$ & $0.4(0.9)$ & $<0.001$ \\
Procalcitonin, mean (SD) & $0.1(0.3)$ & $380.2(187.0,721.2)$ & $<0.001$ \\
Ferritin, median (IQR) & $112.9(26.7,293.4)$ & $47.5(74.5)$ & $<0.001$ \\
Alanine aminotransferase, mean (SD) & $32.5(29.5)$ & $52.0(61.8)$ & $<0.001$ \\
Aspartate aminotransferase, mean (SD) & $26.3(20.9)$ & $91.5(77.5)$ & $<0.001$ \\
Alkaline phosphatase, mean (SD) & $73.3(32.8)$ & $13.1(5.4,20.7)$ & $<0.001$ \\
Troponin, median (IQR) & $3.4(0.4,6.3)$ & & 0.011 \\
\hline IQR: Interquartile Range, SD: Standard deviation & & &
\end{tabular}

When we look at the results of the logistic regression analysis, it was seen that the clinical demographic parameters, age, chronic renal failure and cancer diagnosis, high lactate dehydrogenase and ferritin levels, and low lymphocyte count were the only independent risk factors in predicting the intensive care unit admission of the patients (Table 3 ). The bars showing the relationship between age and gender distribution and the intensive care unit are presented in Figure 1, and the trends of $\mathrm{LDH}$, lymphocyte count and ferritin levels are presented in Figure 2A, 2B, 2C.

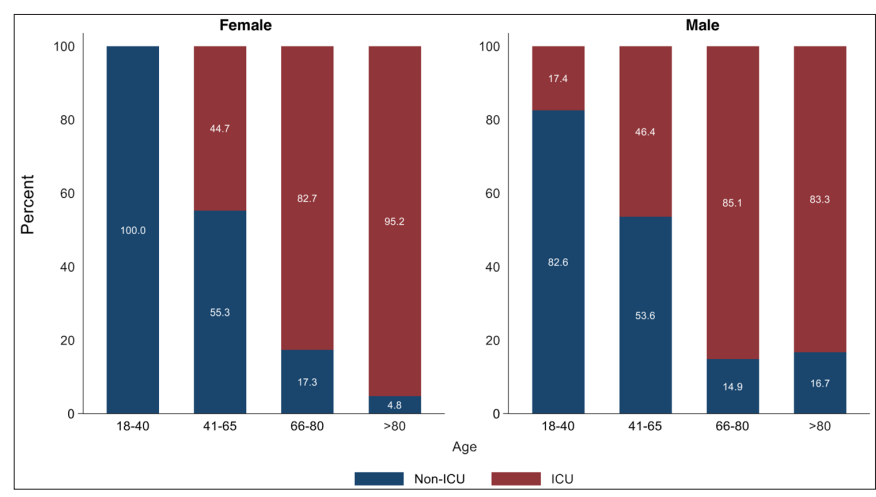

Figure 1. Non-intensive care unit (ICU) vs ICU patients' distributions according to sex and age subgroups

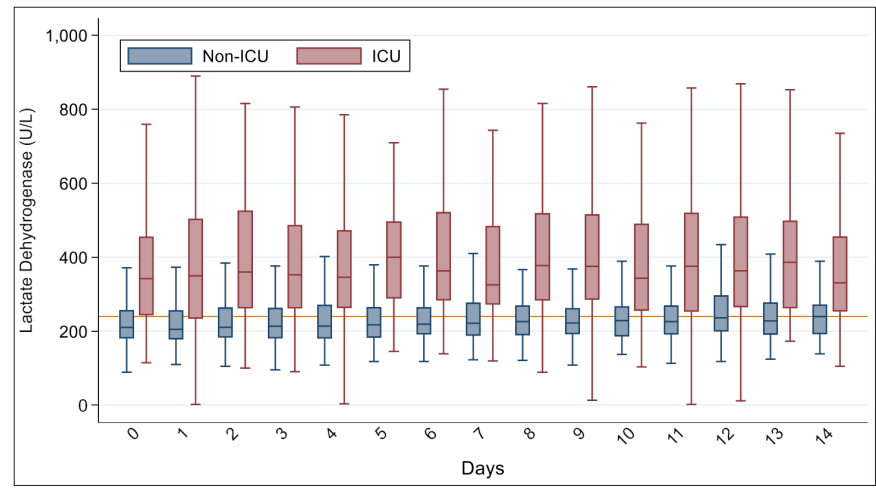

Figure 2A. Dynamic trends of Lactate Dehydrogenase according to Non-intensive care unit (ICU)and ICU patients up to 14-days
Table 3. Predictors of ICU patients according to multivariable logistic regression analysis

\begin{tabular}{|lcc|}
\hline & $\begin{array}{c}\text { Adjusted odds ratio } \\
\text { (95\% confidence interval) }\end{array}$ & p value \\
\hline Age & $1.094(1.077-1.112)$ & $<0.001$ \\
Chronic renal failure & $4.735(1.117-20.067)$ & 0.036 \\
Cancer & $3.957(1.225-12.776)$ & 0.021 \\
Lactate dehydrogenase & $1.006(1.003-1.008)$ & $<0.001$ \\
Lymphocyte & $0.879(0.789-0.980)$ & 0.021 \\
Ferritin & $1.000(1.000-1.001)$ & 0.001 \\
\hline
\end{tabular}

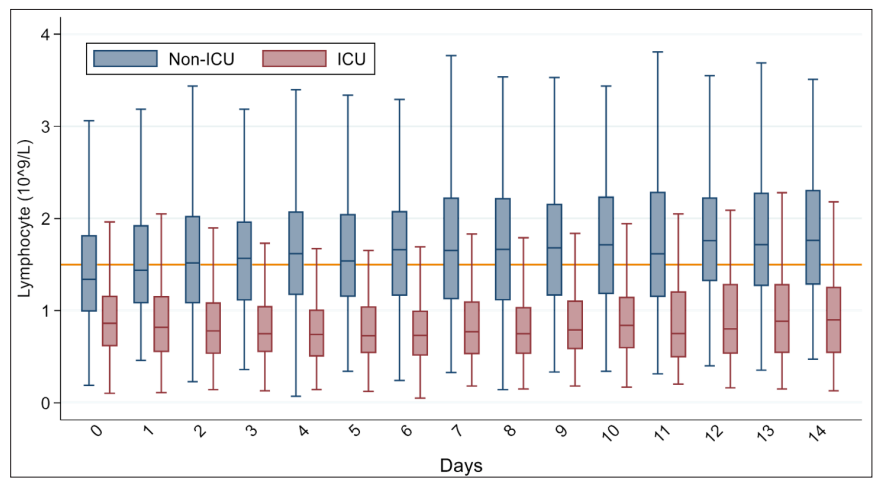

Figure 2B. Dynamic trends of Lymphocyte count according to Nonintensive care unit (ICU) and ICU patients up to 14-days

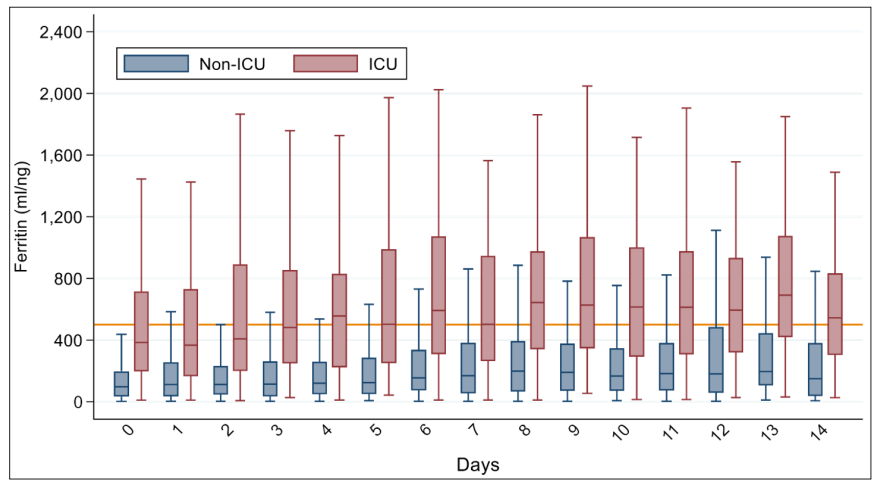

Figure 2C. Dynamic trends of Ferritin according to Non-intensive care unit (ICU) and ICU patients up to 14-days 


\section{DISCUSSION}

According to the results of this study, age, additional morbidities such as chronic kidney failure and cancer, and laboratory data, high lactate dehydrogenase and ferritin and low lymphocyte count alone are independent risks in predicting whether patients who are admitted to the emergency department with the diagnosis of COVID-19 and hospitalized will need intensive care or not. factor was found. The fact that it included a fairly sufficient number of patients and that different parameters were found to be independent risk factors in predicting intensive care admission are the peculiarities of our study.

In the literature, there are different studies on the parameters taken during the intensive care unit admission of COVID-19 patients. In some studies, diabetes and $\mathrm{HbAlc}$ are predictive, and some studies have shown that pneumonia and chronic lung problems are important in ICU admission $(7,8)$. Age is also seen in the studied and published data among these parameters. Many parameters can be used to decide on the hospitalization of patients in adverse situations such as in cases where there are too many patient admissions, the number of beds is insufficient, and the limited usage conditions. Some of these can be added as troponin, lung radiology and clinical findings $(8,9)$. Although clinical findings are at the forefront in intensive care admission, it may be useful to look at additional parameters and make decisions based on independent risk factors in places with limited number of beds. For this reason, the results of our study can support healthcare professionals serving the emergency department in their decision-making and can be used during practical applications in predicting intensive care admission.

In the study of Luo et al. (10), age, neutrophil and platelet count were found to be significantly correlated with CRP and severe disease and poor prognosis. Similarly, in the study of Marin et al. (11), increased age was shown to be associated with lymphopenia and chronic renal failure suffocation, mortality and poor prognosis. In the same study, an increase in $\mathrm{LDH}$, which indicates cellular injury, is associated with mortality and poor prognosis. Covino et al. (12) similar to other studies in the literature, it was shown that advanced age is closely related to intensive care unit admission and mortality. Wu et al. (13) found a close relationship between ferritin level and the development of ARDS in patients with COVID-19 pneumonia. Similarly, Şan et al. (14) found that the complete blood count parameters can help to identify and classify COVID-19 patients into non-severe to severe groups.

In the study conducted by Carlino et al. (5), a significant relationship was found between patients with a history of cancer and admission to intensive care unit. In the study of Hu et al. (15), no significant difference was found between comorbidities (anemia, cancer, hypertension, diabetes, coronary heart disease, chronic obstructive pulmonary disease, cerebral infarction) and serious disease. We attributed this situation to the small number of patients included, unlike our study. Lee et al. (16) in his study, a significant correlation was found between patients with a history of lymphopenia, cancer, and chronic renal failure and mortality. Similarly, Bhargava et al. (17) found a significant relationship between chronic renal failure and severe disease in their study.

This study may have some limitations, one of them being a retrospective study, but the sufficient number of cases is an important factor in closing this gap. The high number of patients in our hospital, the high number of emergency service applications and the large bed capacity can be counted as the strengths of our study.

\section{CONCLUSION}

This study revealed that age, chronic renal failure, and cancer are the only independent risk factors in predicting intensive care admission for patients admitted to the emergency department with the diagnosis of COVID-19, and that high LDH, ferritin levels, and low lymphocyte count are laboratory independent risk factors.

\section{ETHICAL DECLARATIONS}

Ethics Committee Approval: The study was carried out with the permission of Ankara City Hospital No. 2 Clinical Research Ethics Committee (Date: 14.07.2021, Decision No: E2-21-739).

Informed Consent: Because the study was designed retrospectively, no written informed consent form was obtained from patients.

Referee Evaluation Process: Externally peer-reviewed.

Conflict of Interest Statement: The authors have no conflicts of interest to declare.

Financial Disclosure: The authors declared that this study has received no financial support.

Author Contributions: All of the authors declare that they have all participated in the design, execution, and analysis of the paper, and that they have approved the final version.

\section{REFERENCES}

1. Sahin D, Tanacan A, Erol SA, et al. A pandemic center's experience of managing pregnant women with COVID-19 infection in Turkey: A prospective cohort study. Int J Gynecol Obstetr 2020; 151: 74-82.

2. Immovilli P, Morelli N, Antonucci E, Radaelli G, Barbera M, Guidetti D. COVID-19 mortality and ICU admission: the Italian experience. Critical Care 2020; 24: 1. 
3. Walton H, Navaratnam AV, Ormond M, Gandhi V, Mann C. Emergency medicine response to the COVID-19 pandemic in England: a phenomenological study. Emerg Med J 2020; 37: 768-72.

4. Assandri R, Buscarini E, Canetta C, Scartabellati A, Viganò G, Montanelli A. Laboratory biomarkers predicting COVID-19 severity in the emergency room. Arch Med Res 2020; 51: 598-9.

5. Carlino MV, Valenti N, Cesaro F, et al. Predictors of intensive care unit admission in patients with Coronavirus disease 2019 (COVID-19). Monaldi Arch Chest Dis 2020; 9.

6. https://COVID19.saglik.gov.tr/Eklenti/40781/0/COVID9rehberiagirpnomoniardssepsisveseptik sokyontemipdf.pdf

7. Prattichizzo F, de Candia P, Nicolucci A, Ceriello A. Elevated HbAlc levels in pre-COVID-19 infection increases the risk of mortality: a sistematic review and meta-analysis. Diabetes Metab Res Rev 2021: e3476.

8. Zhou Y, Yang Z, Guo Y, et al. A new predictor of disease severity in patients with COVID-19 in Wuhan, China. MedRxiv 2020.03.24.20042119.

9. Nie SF, Yu M, Xie T, et al. Cardiac troponin I is an independent predictor for mortality in hospitalized patients with COVID-19. Circulation 2020; 142: 608-10.

10. Luo X, Zhou W, Yan X, et al. Prognostic value of C-reactive protein in patients with Coronavirus 2019. Clin Infect Dis 2020; 71: 2174-9.

11. Gallo Marin B, Aghagoli G, Lavine K, et al. Predictors of COVID-19 severity: a literature review. Rev Med Virol 2021; 31: $1-10$.

12. Covino M, Sandroni C, Santoro M, et al. Predicting intensive care unit admission and death for COVID-19 patients in the emergency department using early warning scores. Resuscitation 2020; 156: 84-91.

13. Wu C, Chen X, Cai Y, et al. Risk factors associated with acute respiratory distress syndrome and death in patients with Coronavirus disease 2019 pneumonia in Wuhan, China. JAMA Intern Med 2020; 180: 934-43.

14. Şan İ, Gemcioğlu E, Davutoğlu M, et al. Which hematological markers have predictive value as early indicators of severe COVID-19 cases in the emergency department? Turk J Med Sci 2021; 10.3906/sag-2008-6.

15. $\mathrm{Hu} \mathrm{H}, \mathrm{Du} \mathrm{H}, \mathrm{Li}$ J, et al. Early prediction and identification for severe patients during the pandemic of COVID-19: A severe COVID-19 risk model constructed by multivariate logistic regression analysis. J Glob Health 2020; 10: 020510.

16. Lee J, Park SS, Kim TY, Lee DG, Kim DW. Lymphopenia as a biological predictor of outcomes in COVID-19 patients: a nationwide cohort study. Cancers 2021; 26; 13: 471.

17. Bhargava A, Fukushima EA, Levine M, et al. Predictors for severe COVID-19 infection. Clin Infect Dis 2020; 71: 1962-8. 